\title{
Supporting Information for Arylmagnesiation of Alkynes Catalyzed Cooperatively by Iron and Copper Complexes
}

Eiji Shirakawa,* Takafumi Yamagami, Takahiro Kimura, Shigeru Yamaguchi and Tamio Hayashi*

General Remarks. All manipulations of oxygen- and moisture-sensitive materials were conducted with a standard Schlenk technique under a nitrogen atmosphere. Nuclear magnetic resonance spectra were taken on a JEOL JNM LA500 spectrometer $\left({ }^{1} \mathrm{H}, 500 \mathrm{MHz}\right.$; $\left.{ }^{13} \mathrm{C}, 125 \mathrm{MHz}\right)$ spectrometer using tetramethylsilane $\left({ }^{1} \mathrm{H}\right.$ and $\left.{ }^{13} \mathrm{C}\right)$ as an internal standard. GC-MS spectra were taken on Shimazu GCMS-QP5050A. High-resolution mass spectra were obtained with a JEOL JMS-700 spectrometer. Preparative recycling gel permeation chromatography was performed with JAI LC-908 equipped with JAIGEL-1H and -2H using chloroform as an eluent. Unless otherwise noted, reagents were commercially available and used without further purification. Tetrahydrofuran, diethyl ether and benzene were distilled from sodium/benzophenone ketyl. Arylmagnesium bromides were prepared in THF, $\mathrm{Et}_{2} \mathrm{O}$ or $\mathrm{Et}_{2} \mathrm{O}$-benzene $(1: 1)$ by the reaction of the corresponding aryl bromides with magnesium turning.

Iron-Copper-Catalyzed Arylmagnesiation of Alkynes. A General Procedure. A solution of $\mathrm{Fe}(\mathrm{acac})_{3}(7.9 \mathrm{mg}, 22 \mu \mathrm{mol}), \mathrm{CuBr}(6.5 \mathrm{mg}, 45 \mu \mathrm{mol})$ and $\mathrm{PBu}_{3}(45 \mu \mathrm{L}, 36 \mathrm{mg}$, $0.18 \mathrm{mmol})$ in THF $(1.7 \mathrm{~mL})$ was placed in a $20 \mathrm{~mL}$ Schlenk tube and stirred for $10 \mathrm{~min}$ at room temperature. To this solution was added successively an alkyne $(0.45 \mathrm{mmol})$ and a THF, $\mathrm{Et}_{2} \mathrm{O}$ or $\mathrm{Et}_{2} \mathrm{O}-$ benzene $(1: 1)$ solution $(0.33-1.74 \mathrm{M})$ of an arylmagnesium bromide $(0.90$ mmol), and the mixture was stirred at $60{ }^{\circ} \mathrm{C}$. After the time specified in Table 2, water (10 $\mathrm{mL}$ ) was added and the resulting mixture was extracted with diethyl ether $(10 \mathrm{~mL} \times 3)$. The combined organic layer was washed with brine $(10 \mathrm{~mL})$, and dried over anhydrous magnesium sulfate. Evaporation of the solvent followed by purification with PTLC $\left(\mathrm{SiO}_{2}\right)$ gave the corresponding arylmagnesiation products, whose isomer ratio was determined by GC, GC-MS and ${ }^{1} \mathrm{H}$ NMR.

The Reaction of 1 a with $2 a$ in the Presence of a Reduced Amount of $\mathrm{PBu}_{3}$ (Reference 12). A solution of $\mathrm{Fe}(\mathrm{acac})_{3}(4.7 \mathrm{mg}, 13 \mu \mathrm{mol}), \mathrm{CuBr}(3.9 \mathrm{mg}, 27 \mu \mathrm{mol})$ and $\mathrm{PBu}_{3}$ $(11 \mu \mathrm{L}, 9.1 \mathrm{mg}, 45 \mu \mathrm{mol})$ in THF $(0.20 \mathrm{~mL})$ was placed in a $20 \mathrm{~mL}$ Schlenk tube and stirred for $10 \mathrm{~min}$ at room temperature. To this solution was added successively 4-octyne $(50 \mathrm{mg}$, $0.45 \mathrm{mmol})$ and a THF solution $(2.2 \mathrm{M})$ of an arylmagnesium bromide $(0.41 \mathrm{~mL}, 0.90 \mathrm{mmol})$, and the mixture was stirred at $65{ }^{\circ} \mathrm{C}$ for $48 \mathrm{~h}$. Water $(10 \mathrm{~mL})$ was added and the resulting mixture was extracted with diethyl ether $(10 \mathrm{~mL}$ x 3$)$. The combined organic layer was washed with brine $(10 \mathrm{~mL})$, and dried over anhydrous magnesium sulfate. Evaporation of the solvent followed by purification with PTLC $\left(\mathrm{SiO}_{2}\right.$, hexane) gave 3a $(59 \mathrm{mg}, 61 \%, E: Z=$ 91:9). 
The spectral data of the arylmagnesiation products are as follows. For ${ }^{1} \mathrm{H}$ and ${ }^{13} \mathrm{C}$ NMR, only the data of the major $(E)$-isomers are given. Elemental analysis was conducted for mixtures of stereo- and regioisomers.

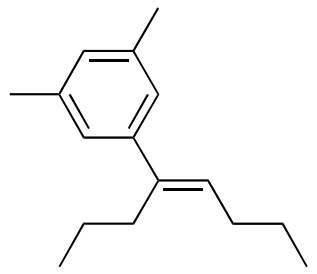

3a: entry 1 in Table 1

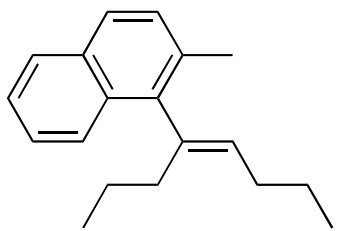

3b: entry 4 in Table 1

(E)-4-(3,5-Dimethylphenyl)-4-octene (3a). A colorless oil. ${ }^{1} \mathrm{H}$ NMR $\left(500 \mathrm{MHz}, \mathrm{CDCl}_{3}\right) \delta 0.88(\mathrm{t}, J=7.4 \mathrm{~Hz}, 3 \mathrm{H}), 0.95(\mathrm{t}, J=7.4 \mathrm{~Hz}, 3 \mathrm{H})$, 1.35 (sext, $J=7.5 \mathrm{~Hz}, 2 \mathrm{H}$ ), 1.46 (sext, $J=7.4 \mathrm{~Hz}, 2 \mathrm{H}$ ), 2.15 (q, $J=$ $7.4 \mathrm{~Hz}, 2 \mathrm{H}), 2.30$ (s, $6 \mathrm{H}), 2.44$ (t, $J=7.7 \mathrm{~Hz}, 2 \mathrm{H}), 5.61(\mathrm{t}, J=7.3 \mathrm{~Hz}$, $1 \mathrm{H}), 6.85$ (s, $1 \mathrm{H}), 6.94(\mathrm{~s}, 2 \mathrm{H}) .{ }^{13} \mathrm{C} \mathrm{NMR}\left(125 \mathrm{MHz}, \mathrm{CDCl}_{3}\right) \delta 13.95$, 13.98, 21.4, 21.9, 23.1, 30.6, 31.8, 124.3, 128.1, 128.8, 137.4, 140.2, 143.6. Anal. Calcd for $\mathrm{C}_{16} \mathrm{H}_{24}$ : C, 88.82; H, 11.18. Found: C, 89.11; H, 11.28.

(E)-4-(2-Methylnaphth-1-yl)-4-octene (3b). A colorless oil. ${ }^{1} \mathrm{H}$ NMR $\left(500 \mathrm{MHz}, \mathrm{CDCl}_{3}\right) \delta 0.86(\mathrm{t}, J=7.4 \mathrm{~Hz}, 3 \mathrm{H}), 1.02(\mathrm{t}, J=7.4 \mathrm{~Hz}, 3 \mathrm{H})$, $1.23-1.39$ (m, $2 \mathrm{H}), 1.53$ (sext, $J=7.4 \mathrm{~Hz}, 2 \mathrm{H}), 2.32$ (q, $J=7.3 \mathrm{~Hz}, 2$ H), 2.37-2.46 (m, $2 \mathrm{H}), 2.42(\mathrm{~s}, 3 \mathrm{H}), 5.35$ (t, $J=7.3 \mathrm{~Hz}, 1 \mathrm{H}), 7.31(\mathrm{~d}$, $J=8.4 \mathrm{~Hz}, 1 \mathrm{H}), 7.33-7.42(\mathrm{~m}, 2 \mathrm{H}), 7.64(\mathrm{~d}, J=8.3 \mathrm{~Hz}, 1 \mathrm{H}), 7.76(\mathrm{~d}$, $J=7.7 \mathrm{~Hz}, 1 \mathrm{H}), 7.89(\mathrm{~d}, J=8.2 \mathrm{~Hz}, 1 \mathrm{H}) .{ }^{13} \mathrm{C} \mathrm{NMR}(125 \mathrm{MHz}$, $\left.\mathrm{CDCl}_{3}\right) \delta 14.0,14.7,20.5,21.7,23.1,30.2,35.3,124.4,125.3,126.19,126.24,127.8,128.7$, 131.4, 132.1, 132.3, 132.6, 137.7, 140.7. Anal. Calcd for $\mathrm{C}_{19} \mathrm{H}_{24}$ : C, 90.42; H, 9.58. Found: $\quad$ C, $90.69 ; \mathrm{H}, 9.84$.

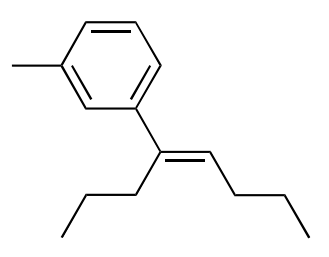

entry 3 in Table 2

(E)-4-(3-Methylphenyl)-4-octene. A colorless oil. ${ }^{1} \mathrm{H}$ NMR $(500 \mathrm{MHz}$, $\left.\mathrm{CDCl}_{3}\right) \delta 0.88(\mathrm{t}, J=7.4 \mathrm{~Hz}, 3 \mathrm{H}), 0.96(\mathrm{t}, J=7.4 \mathrm{~Hz}, 3 \mathrm{H}), 1.36$ (sext, $J$ $=7.5 \mathrm{~Hz}, 2 \mathrm{H}), 1.46$ (sext, $J=7.4 \mathrm{~Hz}, 2 \mathrm{H}), 2.16(\mathrm{q}, J=7.4 \mathrm{~Hz}, 2 \mathrm{H})$, $2.34(\mathrm{~s}, 3 \mathrm{H}), 2.46(\mathrm{t}, J=7.7 \mathrm{~Hz}, 2 \mathrm{H}), 5.63(\mathrm{t}, J=7.3 \mathrm{~Hz}, 1 \mathrm{H}), 7.02(\mathrm{~d}, J$ $=7.2 \mathrm{~Hz}, 1 \mathrm{H}), 7.12(\mathrm{~d}, J=8.1 \mathrm{~Hz}, 1 \mathrm{H}), 7.14(\mathrm{~s}, 1 \mathrm{H}), 7.17(\mathrm{t}, J=7.6 \mathrm{~Hz}$, $31.8,123.5,127.11,127.14,128.0,129.0,137.5,140.2,143.6$. Anal. Calcd for $\mathrm{C}_{15} \mathrm{H}_{22}$ : C, 89.04; H, 10.96. Found: C, 89.31; H, 10.86 .

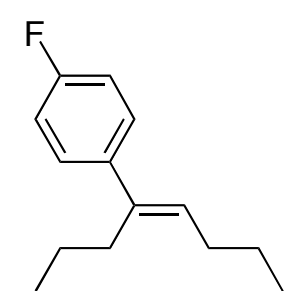

entry 6 in Table 2

(E)-4-(4-Fluorophenyl)-4-octene. A colorless oil. ${ }^{1} \mathrm{H}$ NMR $(500 \mathrm{MHz}$, $\left.\mathrm{CDCl}_{3}\right) \delta 0.87(\mathrm{t}, J=7.4 \mathrm{~Hz}, 3 \mathrm{H}), 0.96(\mathrm{t}, J=7.4 \mathrm{~Hz}, 3 \mathrm{H}), 1.34(\operatorname{sext}, J$ $=7.5 \mathrm{~Hz}, 2 \mathrm{H}), 1.46(\mathrm{sext}, J=7.4 \mathrm{~Hz}, 2 \mathrm{H}), 2.16(\mathrm{q}, J=7.4 \mathrm{~Hz}, 2 \mathrm{H})$, $2.44(\mathrm{t}, J=7.6 \mathrm{~Hz}, 2 \mathrm{H}), 5.59(\mathrm{t}, J=7.2 \mathrm{~Hz}, 1 \mathrm{H}), 6.97(\mathrm{t}, J=8.8 \mathrm{~Hz}, 2$ H) , 7.28 (dd, $J=8.7,5.6 \mathrm{~Hz}, 2 \mathrm{H}) .{ }^{13} \mathrm{C} \mathrm{NMR}\left(125 \mathrm{MHz}, \mathrm{CDCl}_{3}\right) \delta 13.89$, 13.92, 21.7, 23.0, 30.6, 31.8, $114.8\left({ }^{2} J_{\mathrm{C}-\mathrm{F}}=19.9 \mathrm{~Hz}\right), 127.8\left({ }^{3} J_{\mathrm{C}-\mathrm{F}}=7.8\right.$ $\mathrm{Hz}), 129.2,139.1,139.6\left({ }^{4} J_{\mathrm{C}-\mathrm{F}}=3.1 \mathrm{~Hz}\right), 161.7\left({ }^{1} J_{\mathrm{C}-\mathrm{F}}=239.9 \mathrm{~Hz}\right)$. HRMS (EI) Calcd for $\mathrm{C}_{14} \mathrm{H}_{19} \mathrm{~F}: \quad \mathrm{M}^{+}, 206.1471$. Found: $m / z$ 206.1475. 


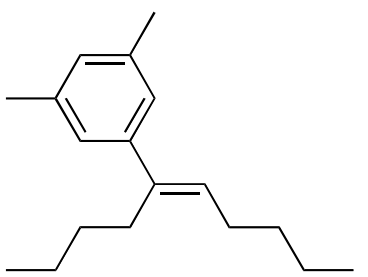

entry 7 in Table 2

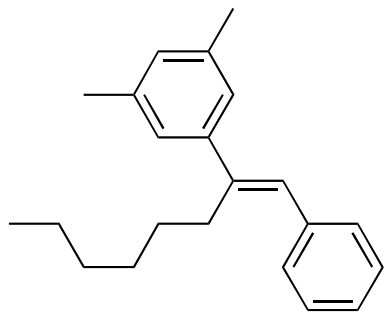

entry 8 in Table 2

(E)-5-(3,5-Dimethylphenyl)-5-decene. A colorless oil. ${ }^{1} \mathrm{H}$ NMR (500 $\left.\mathrm{MHz}, \mathrm{CDCl}_{3}\right) \delta 0.87(\mathrm{t}, J=7.0 \mathrm{~Hz}, 3 \mathrm{H}), 0.92(\mathrm{t}, J=7.0 \mathrm{~Hz}, 3 \mathrm{H})$, 1.25-1.45 (m, $8 \mathrm{H}), 2.17(\mathrm{q}, J=7.1 \mathrm{~Hz}, 2 \mathrm{H}), 2.30(\mathrm{~s}, 6 \mathrm{H}), 2.45(\mathrm{t}, J$ $=7.0 \mathrm{~Hz}, 2 \mathrm{H}), 5.59(\mathrm{t}, J=7.2 \mathrm{~Hz}, 1 \mathrm{H}), 6.85(\mathrm{~s}, 1 \mathrm{H}), 6.94(\mathrm{~s}, 2 \mathrm{H})$. ${ }^{13} \mathrm{C}$ NMR $\left(125 \mathrm{MHz}, \mathrm{CDCl}_{3}\right) \delta 13.97,14.04,21.4,22.5,22.7,28.2$, 29.6, 31.0, 32.2, 124.3, 128.0, 128. 7, 137.4, 140.3, 143.7. Anal. Calcd for $\mathrm{C}_{18} \mathrm{H}_{28}$ : C, 88.45; H, 11.55. Found: C, 88.20; H, 11.36.

(E)-2-(3,5-Dimethylphenyl)-1-phenyl-1-octene. A colorless oil. ${ }^{1} \mathrm{H}$ $\operatorname{NMR}\left(500 \mathrm{MHz}, \mathrm{CDCl}_{3}\right) \delta 0.84(\mathrm{t}, J=6.8 \mathrm{~Hz}, 3 \mathrm{H}), 1.17-1.36(\mathrm{~m}, 6$ H), 1.37-1.46 (m, $2 \mathrm{H}), 2.34(\mathrm{~s}, 6 \mathrm{H}), 2.66(\mathrm{t}, J=7.8 \mathrm{~Hz}, 2 \mathrm{H}), 6.65$ $(\mathrm{s}, 1 \mathrm{H}), 6.93(\mathrm{~s}, 1 \mathrm{H}), 7.06(\mathrm{~s}, 2 \mathrm{H}), 7.22(\mathrm{t}, J=6.7 \mathrm{~Hz}, 1 \mathrm{H}), 7.30(\mathrm{~d}$, $J=7.5 \mathrm{~Hz}, 2 \mathrm{H}), 7.34(\mathrm{t}, J=7.5 \mathrm{~Hz}, 2 \mathrm{H}) .{ }^{13} \mathrm{C} \mathrm{NMR}(125 \mathrm{MHz}$, $\left.\mathrm{CDCl}_{3}\right) \delta 14.0,21.4,22.6,28.7,29.4,30.3,31.5,124.5,126.4,127.7$, 128.2, 128.78, 128.82, 137.7, 138.5, 143.3, 143.7. Anal. Calcd for $\mathrm{C}_{22} \mathrm{H}_{28}$ : C, 90.35; H, 9.65. Found: C, 90.63; H, 9.72.

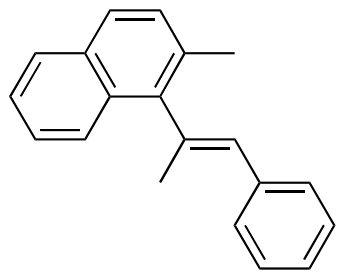

entry 9 in Table 2

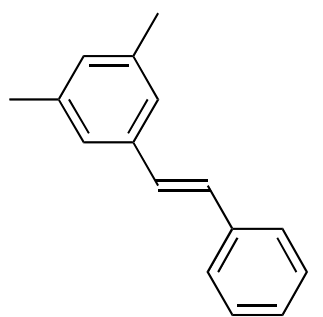

entry 10 in Table 2

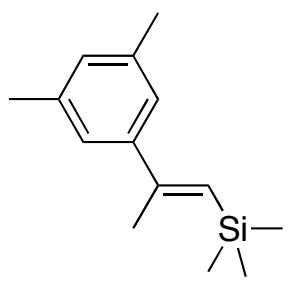

entry 11 in Table 2

(E)-4-(2-Methylnaphth-1-yl)-1-phenyl-1-propene. A colorless oil. ${ }^{1} \mathrm{H}$ NMR $\left(500 \mathrm{MHz}, \mathrm{CDCl}_{3}\right) \delta 2.24(\mathrm{~s}, 3 \mathrm{H}), 2.48(\mathrm{~s}, 3 \mathrm{H}), 6.45(\mathrm{~s}, 1 \mathrm{H})$, $7.28(\mathrm{t}, J=7.3 \mathrm{~Hz}, 1 \mathrm{H}), 7.37(\mathrm{~d}, J=8.5 \mathrm{~Hz}, 1 \mathrm{H}), 7.39-7.50(\mathrm{~m}, 6 \mathrm{H})$, $7.70(\mathrm{~d}, J=8.4 \mathrm{~Hz}, 1 \mathrm{H}), 7.81(\mathrm{~d}, J=7.7 \mathrm{~Hz}, 1 \mathrm{H}), 7.92(\mathrm{~d}, J=8.0 \mathrm{~Hz}$, $1 \mathrm{H}) .{ }^{13} \mathrm{C}$ NMR $\left(125 \mathrm{MHz}, \mathrm{CDCl}_{3}\right) \delta 20.0,124.9,125.6,126.1,126.7$, 126.8, 128.1, 128.4, 128.9, 129.1, 130.6, 131.7, 131.8, 132.3, 137.0, 138.1, 141.5. Anal. Calcd for $\mathrm{C}_{22} \mathrm{H}_{28}$ : C, 92.98; H, 7.02. Found: C, 92.98; H, 7.02. Found: C, 92.75; H, 7.24.

(E)-1-(3,5-Dimethylphenyl)-2-phenylethene. A colorless oil. ${ }^{1} \mathrm{H}$ NMR $\left(500 \mathrm{MHz}, \mathrm{CDCl}_{3}\right) \delta 2.36(\mathrm{~s}, 6 \mathrm{H}), 6.94(\mathrm{~s}, 1 \mathrm{H}), 7.07(\mathrm{~d}, J=16.3 \mathrm{~Hz}, 1$ H), $7.12(\mathrm{~d}, J=16.3 \mathrm{~Hz}, 1 \mathrm{H}), 7.17(\mathrm{~s}, 2 \mathrm{H}), 7.27$ (t, $J=7.4 \mathrm{~Hz}, 1 \mathrm{H})$, $7.37(\mathrm{t}, J=7.5 \mathrm{~Hz}, 2 \mathrm{H}), 7.52(\mathrm{~d}, J=7.2 \mathrm{~Hz}, 2 \mathrm{H}) .{ }^{13} \mathrm{C} \mathrm{NMR}(125 \mathrm{MHz}$, $\left.\mathrm{CDCl}_{3}\right) \delta 21.3,124.4,126.4,127.4,128.3,128.6,128.9,129.4,137.2$, 137.5, 138.1. Anal. Calcd for $\mathrm{C}_{16} \mathrm{H}_{16}$ : C, 92.26; H, 7.74. Found: C, 92.48; H, 7.95.

(E)-2-(3,5-Dimethylphenyl)-1-(trimethylsilyl)-1-propene. A colorless oil. ${ }^{1} \mathrm{H}$ NMR (500 MHz, $\mathrm{CDCl}_{3}$ ) $\delta 0.17$ (s, $9 \mathrm{H}$ ), 2.17 (s, $\left.3 \mathrm{H}\right), 2.30$ (s, 6 $\mathrm{H}), 5.85(\mathrm{~s}, 1 \mathrm{H}), 6.89(\mathrm{~s}, 1 \mathrm{H}), 7.05(\mathrm{~s}, 2 \mathrm{H}) .{ }^{13} \mathrm{C} \mathrm{NMR}(125 \mathrm{MHz}$, $\left.\mathrm{CDCl}_{3}\right) \delta 0.03,21.0,21.4,123.4,126.9,128.9,137.5,144.6,152.0$. Anal. Calcd for $\mathrm{C}_{14} \mathrm{H}_{22} \mathrm{Si}$ : C, 76.99; $\mathrm{H}, 10.15$. Found (as a mixture of isomers): $\mathrm{C}, 77.04 ; \mathrm{H}, 10.21$. Found (as a pure isomer): $\mathrm{C}, 77.08 ; \mathrm{H}$, 10.00 . 
Configuration of some of the arylmagnesiation products were determined by NOE experiments in ${ }^{1} \mathrm{H}$ NMR as shown below.

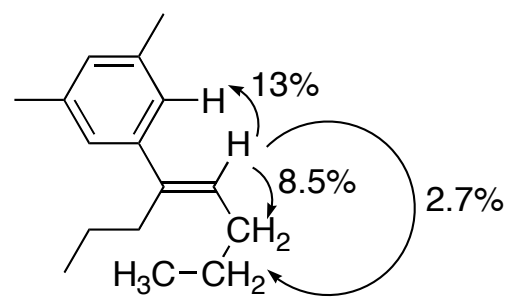

$(E)-3 a$

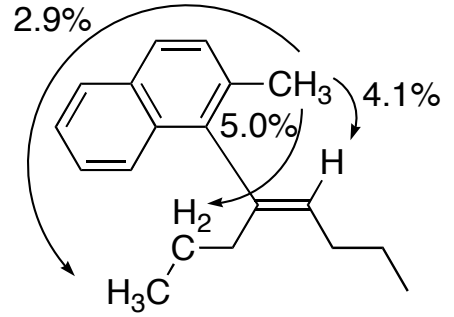

$(E)-\mathbf{3 b}$

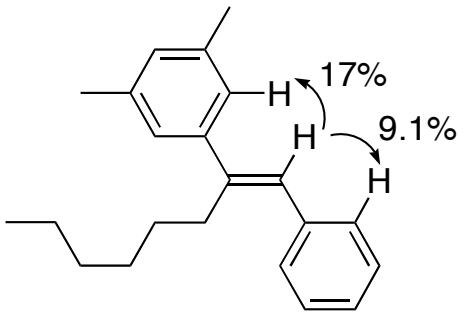

(E)-2-(3,5-Dimethylphenyl)1-phenyl-1-octene

Other arylmagnesiation products listed in Table 2 have already been reported in the literature. Their ${ }^{1} \mathrm{H}$ NMR data are as follows.

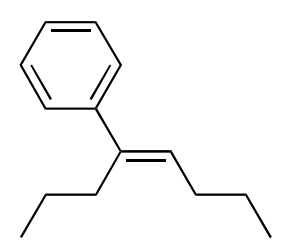

entry 1 in Table 2

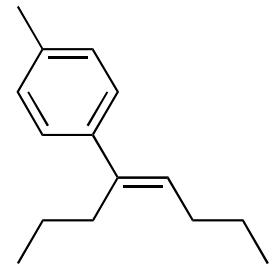

entry 2 in Table 2

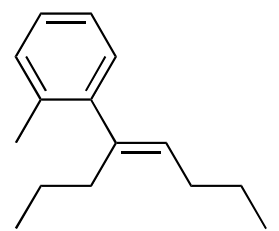

entry 4 in Table 2

(E)-4-Phenyl-4-octene. ${ }^{1}$ A colorless oil. ${ }^{1} \mathrm{H}$ NMR $\left(500 \mathrm{MHz}, \mathrm{CDCl}_{3}\right) \delta$ $0.88(\mathrm{t}, J=7.3 \mathrm{~Hz}, 3 \mathrm{H}), 0.96(\mathrm{t}, J=7.4 \mathrm{~Hz}, 3 \mathrm{H}), 1.36$ (sext, $J=7.5 \mathrm{~Hz}, 2$ H), 1.47 (sext, $J=7.4 \mathrm{~Hz}, 2 \mathrm{H}), 2.17$ (q, $J=7.4 \mathrm{~Hz}, 2 \mathrm{H}), 2.47$ (t, $J=7.6$ $\mathrm{Hz}, 2 \mathrm{H}), 5.65(\mathrm{t}, J=7.3 \mathrm{~Hz}, 1 \mathrm{H}), 7.20(\mathrm{t}, J=7.2 \mathrm{~Hz}, 1 \mathrm{H}), 7.28(\mathrm{t}, J=7.6$ $\mathrm{Hz}, 2 \mathrm{H}), 7.33(\mathrm{~d}, J=8.0 \mathrm{~Hz}, 2 \mathrm{H})$.

(E)-4-(4-Methylphenyl)-4-octene. ${ }^{1}$ A colorless oil. ${ }^{1} \mathrm{H}$ NMR $(500 \mathrm{MHz}$, $\left.\mathrm{CDCl}_{3}\right) \delta 0.86(\mathrm{t}, J=7.4 \mathrm{~Hz}, 3 \mathrm{H}), 0.94(\mathrm{t}, J=7.4 \mathrm{~Hz}, 3 \mathrm{H}), 1.34$ (sext, $J=$ $7.5 \mathrm{~Hz}, 2 \mathrm{H}), 1.44$ (sext, $J=7.4 \mathrm{~Hz}, 2 \mathrm{H}), 2.14$ (q, $J=7.4 \mathrm{~Hz}, 2 \mathrm{H}), 2.31$ (s, $3 \mathrm{H}), 2.44(\mathrm{t}, J=7.7 \mathrm{~Hz}, 2 \mathrm{H}), 5.61(\mathrm{t}, J=7.3 \mathrm{~Hz}, 1 \mathrm{H}), 7.09(\mathrm{~d}, J=8.3 \mathrm{~Hz}$, $2 \mathrm{H}), 7.21(\mathrm{~d}, J=8.3 \mathrm{~Hz}, 2 \mathrm{H})$.

(E)-4-(2-Methylphenyl)-4-octene. ${ }^{2}$ A colorless oil. ${ }^{1} \mathrm{H}$ NMR $(500 \mathrm{MHz}$, $\left.\mathrm{CDCl}_{3}\right) \delta 0.86(\mathrm{t}, J=7.4 \mathrm{~Hz}, 3 \mathrm{H}), 0.95(\mathrm{t}, J=7.4 \mathrm{~Hz}, 3 \mathrm{H}), 1.28$ (sext, $J=$ $7.5 \mathrm{~Hz}, 2 \mathrm{H}$ ), 1.44 (sext, $J=7.4 \mathrm{~Hz}, 2 \mathrm{H}$ ), 2.15 (q, $J=7.4 \mathrm{~Hz}, 2 \mathrm{H}$ ), 2.26 (s, $3 \mathrm{H}), 2.30(\mathrm{t}, J=7.8 \mathrm{~Hz}, 2 \mathrm{H}), 5.24(\mathrm{t}, J=7.3 \mathrm{~Hz}, 1 \mathrm{H}), 7.01-7.05(\mathrm{~m}, \quad 1$ $\mathrm{H}), 7.07-7.16(\mathrm{~m}, 3 \mathrm{H})$.

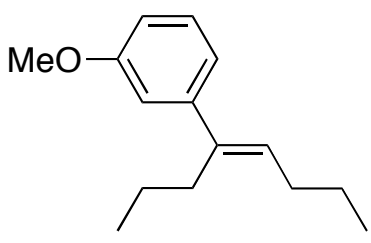

entry 5 in Table 2
(E)-4-(3-Methoxyphenyl)-4-octene. ${ }^{2}$ A colorless oil. ${ }^{1} \mathrm{H}$ NMR (500 $\left.\mathrm{MHz}, \mathrm{CDCl}_{3}\right) \delta 0.86(\mathrm{t}, J=7.4 \mathrm{~Hz}, 3 \mathrm{H}), 0.94(\mathrm{t}, J=7.4 \mathrm{~Hz}, 3 \mathrm{H})$, 1.35 (sext, $J=7.5 \mathrm{~Hz}, 2 \mathrm{H}$ ), 1.45 (sext, $J=7.4 \mathrm{~Hz}, 2 \mathrm{H}$ ), 2.15 (q, $J=$ $7.3 \mathrm{~Hz}, 2 \mathrm{H}), 2.44(\mathrm{t}, J=7.7 \mathrm{~Hz}, 2 \mathrm{H}), 3.80(\mathrm{~s}, 3 \mathrm{H}), 5.65(\mathrm{t}, J=7.3$ $\mathrm{Hz}, 1 \mathrm{H}), 6.73-6.77(\mathrm{~m}, 1 \mathrm{H}), 6.86-6.88(\mathrm{~m}, 1 \mathrm{H}), 6.90-6.94(\mathrm{~m}, 1$ $\mathrm{H}), 7.19(\mathrm{t}, J=7.9 \mathrm{~Hz}, 1 \mathrm{H})$. 
Arylmagnesiation of $2 a$ with $1 b$ Followed by the Reaction with Benzaldehyde (Scheme 1). A solution of $\mathrm{Fe}(\mathrm{acac})_{3}(2.6 \mathrm{mg}, 7.4 \mu \mathrm{mol}), \mathrm{CuBr}(2.2 \mathrm{mg}, 15 \mu \mathrm{mol})$ and $\mathrm{PBu}_{3}$ $(12 \mathrm{mg}, 0.59 \mathrm{mmol})$ in THF $(0.60 \mathrm{~mL})$ was placed in a $20 \mathrm{~mL}$ Schlenk tube and stirred for 10 min at room temperature. To this solution was added successively 4-octyne (2a: $16.5 \mathrm{mg}$, $0.150 \mathrm{mmol})$ and a $\mathrm{Et}_{2} \mathrm{O}$-benzene solution $(0.33 \mathrm{M})$ of 2-methylnaphth-1-ylmagnesium bromide $(\mathbf{1 b}: 0.91 \mathrm{~mL}, 0.30 \mathrm{mmol})$, and the mixture was stirred at $60{ }^{\circ} \mathrm{C}$ for $24 \mathrm{~h}$. Then benzaldehyde $(31.8 \mathrm{mg}, 0.300 \mathrm{mmol})$ was added at $0{ }^{\circ} \mathrm{C}$ and the mixture was stirred for $2 \mathrm{~h}$. After addition of water $(10 \mathrm{~mL})$, the resulting mixture was extracted with diethyl ether $(10$ $\mathrm{mL} \times 3)$. The combined organic layer was washed with brine $(10 \mathrm{~mL})$, and dried over anhydrous magnesium sulfate. Evaporation of the solvent followed by purification with PTLC $\left(\mathrm{SiO}_{2}\right)$ gave two separated diastereomers (4a: $\left.22.2 \mathrm{mg}, 43 \% ; 4 \mathbf{b}: 12.0 \mathrm{mg}, 23 \%\right)$ of (Z)-3-(2-methylnaphth-1-yl)-1-phenyl-2-propylhex-2-en-1-ol as colorless oils.

4a. $\left({ }^{1} \mathrm{H} 500 \mathrm{MHz}, \mathrm{CDCl}_{3}\right) \delta 0.82(\mathrm{t}, J=7.3 \mathrm{~Hz}, 3 \mathrm{H}), 0.88(\mathrm{t}, J=7.3 \mathrm{~Hz}, 3 \mathrm{H}), 1.04-1.15(\mathrm{~m}$, $1 \mathrm{H}), 1.15-1.25(\mathrm{~m}, 1 \mathrm{H}), 1.28-1.41(\mathrm{~m}, 1 \mathrm{H}), 1.53(\mathrm{~d}, J=3.0 \mathrm{~Hz}, 1 \mathrm{H}), 1.50-1.63(\mathrm{~m}, 1 \mathrm{H})$, 2.14-2.23 (m, $3 \mathrm{H}), 2.40$ (s, $3 \mathrm{H}), 2.50-2.62(\mathrm{~m}, 1 \mathrm{H}), 4.92(\mathrm{~d}, J=3.0 \mathrm{~Hz}, 1 \mathrm{H}), 7.12-7.20(\mathrm{~m}$, $3 \mathrm{H}), 7.20-7.27(\mathrm{~m}, 2 \mathrm{H}), 7.36(\mathrm{~d}, J=8.4 \mathrm{~Hz}, 1 \mathrm{H}), 7.41(\mathrm{t}, J=7.4 \mathrm{~Hz}, 1 \mathrm{H}), 7.48(\mathrm{t}, J=6.9$ $\mathrm{Hz}, 1 \mathrm{H}), 7.70(\mathrm{~d}, J=8.4 \mathrm{~Hz}, 1 \mathrm{H}), 7.81(\mathrm{~d}, J=7.9 \mathrm{~Hz}, 1 \mathrm{H}), 8.00(\mathrm{~d}, J=8.4 \mathrm{~Hz}, 1 \mathrm{H}) .{ }^{13} \mathrm{C}$ NMR $\left(125 \mathrm{MHz}, \mathrm{CDCl}_{3}\right)$ : $\delta 14.9,15.0,20.6,21.5,24.1,29.7,36.6,74.2,124.9,125.4$, 125.8, 126.2, 126.7, 126.8, 127.9, 128.4, 128.9, 132.2, 132.7, 132.8, 136.8, 137.7, 139.7, 142.6. Anal. Calcd for $\mathrm{C}_{26} \mathrm{H}_{30} \mathrm{O}$ : C, 87.10; H, 8.43. Found: C, 86.99; H, 8.49.

4b. ${ }^{1} \mathrm{H}$ NMR $\left(500 \mathrm{MHz}, \mathrm{CDCl}_{3}\right) \delta 0.82(\mathrm{t}, J=7.3 \mathrm{~Hz}, 3 \mathrm{H}), 0.87(\mathrm{t}, J=7.3 \mathrm{~Hz}, 3 \mathrm{H})$, 0.95-1.08 (m, $1 \mathrm{H}), 1.21-1.38(\mathrm{~m}, 2 \mathrm{H}), 1.60$ (bs, $1 \mathrm{H}), 1.51-1.63(\mathrm{~m}, 1 \mathrm{H}), 2.18$ (td, $J=12.5$, $4.9 \mathrm{~Hz}, 1 \mathrm{H}), 2.25-2.39(\mathrm{~m}, 2 \mathrm{H}), 2.46-2.56(\mathrm{~m}, 1 \mathrm{H}), 2.53(\mathrm{~s}, 3 \mathrm{H}), 5.07(\mathrm{~s}, 1 \mathrm{H}), 7.03$ (d, $J=$ $6.4 \mathrm{~Hz}, 2 \mathrm{H}), 7.07-7.20(\mathrm{~m}, 3 \mathrm{H}), 7.37$ (d, $J=8.4 \mathrm{~Hz}, 1 \mathrm{H}), 7.38-7.47$ (m, $2 \mathrm{H}), 7.69$ (d, $J=$ $8.4 \mathrm{~Hz}, 1 \mathrm{H}), 7.75(\mathrm{~d}, J=7.4 \mathrm{~Hz}, 1 \mathrm{H}), 7.81(\mathrm{~d}, J=7.0 \mathrm{~Hz}, 1 \mathrm{H}) .{ }^{13} \mathrm{C}$ NMR $(125 \mathrm{MHz}$, $\left.\mathrm{CDCl}_{3}\right): \quad \delta 14.8,15.0,20.9,21.5,24.1,29.5,37.0,74.6,124.9,125.6,125.8,126.4,126.7$, $126.8,127.8,128.2,128.7,132.2,132.3,132.8,137.2,137.5,139.4,142.8$. Anal. Calcd for $\mathrm{C}_{26} \mathrm{H}_{30} \mathrm{O}: \quad \mathrm{C}, 87.10 ; \mathrm{H}, 8.43$. Found: C, 87.02; H, 8.73.

Both of isomers $\mathbf{4 a}$ and $\mathbf{4 b}$ were determined to have ( $Z$ )-configuration on the basis of NOE studies as shown below. They must be diastereomers due to the central chirality at the $\alpha$-carbon of the allylic alcohol and the axial chirality based on the restricted rotation about the naphthyl-alkenyl bond.

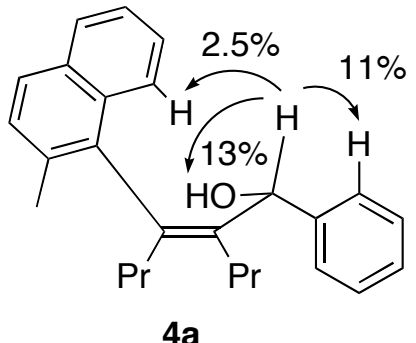

$4 a$

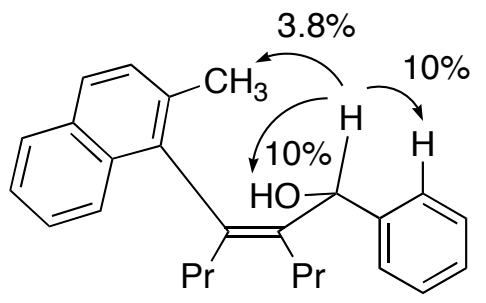

$4 b$ 
Arylmagnesiation of $2 a$ with $1 b$ Followed by the Reaction with Benzyl Bromide (Scheme 1). A solution of $\mathrm{Fe}(\mathrm{acac})_{3}(5.3 \mathrm{mg}, 14 \mu \mathrm{mol}), \mathrm{CuBr}(4.3 \mathrm{mg}, 30 \mu \mathrm{mol})$ and $\mathrm{PBu}_{3}(24$ $\mathrm{mg}, 0.12 \mathrm{mmol})$ in THF $(1.0 \mathrm{~mL})$ was placed in a $20 \mathrm{~mL}$ Schlenk tube and stirred for $10 \mathrm{~min}$ at room temperature. To this solution was added successively 4-octyne (2a: $32.0 \mathrm{mg}, 0.290$ $\mathrm{mmol}$ ) and a $\mathrm{Et}_{2} \mathrm{O}$-benzene solution $(0.33 \mathrm{M})$ of 2-methylnaphth-1-ylmagnesium bromide (1b: $1.8 \mathrm{~mL}, 0.60 \mathrm{mmol}$ ), and the mixture was stirred at $60{ }^{\circ} \mathrm{C}$ for $18 \mathrm{~h}$. Then benzyl bromide (100 mg, $0.58 \mathrm{mmol}$ ) was added at $0{ }^{\circ} \mathrm{C}$ and the mixture was stirred for $1.5 \mathrm{~h}$. After addition of water $(10 \mathrm{~mL})$, the resulting mixture was extracted with diethyl ether $(10 \mathrm{~mL} \times 3)$. The combined organic layer was washed with brine $(10 \mathrm{~mL})$, and dried over anhydrous magnesium sulfate. Evaporation of the solvent followed by purification by GPC gave (Z)-4-benzyl-5-(2-methylnaphth-1-yl)-4-octene (5: $49 \mathrm{mg}, 49 \%)$ as a colorless oil. ${ }^{1} \mathrm{H}$ NMR $\left(500 \mathrm{MHz}, \mathrm{CDCl}_{3}\right) \delta 0.81(\mathrm{t}, J=7.3 \mathrm{~Hz}, 3 \mathrm{H}), 0.99(\mathrm{t}, J=7.3 \mathrm{~Hz}, 3 \mathrm{H}), 1.24-1.37(\mathrm{~m}, 2 \mathrm{H})$, 1.48-1.64 (m, 2 H), 2.18-2.34 (m, 2 H), 2.38-2.48 (m, 2 H), 2.39 (s, $3 \mathrm{H}), 3.02$ (d, J = 14.8 Hz, $1 \mathrm{H}), 3.08$ (d, $J=14.8 \mathrm{~Hz}, 1 \mathrm{H}), 6.89$ (d, $J=7.2 \mathrm{~Hz}, 2 \mathrm{H}), 7.06$ (t, $J=7.3 \mathrm{~Hz}, 1 \mathrm{H}), 7.11$ $(\mathrm{t}, J=7.3 \mathrm{~Hz}, 2 \mathrm{H}), 7.32(\mathrm{~d}, J=8.3 \mathrm{~Hz}, 1 \mathrm{H}), 7.38(\mathrm{t}, J=8.0 \mathrm{~Hz}, 1 \mathrm{H}), 7.43(\mathrm{t}, J=7.6 \mathrm{~Hz}, 1$ $\mathrm{H}), 7.65(\mathrm{~d}, J=8.3 \mathrm{~Hz}, 1 \mathrm{H}), 7.77(\mathrm{~d}, J=8.0 \mathrm{~Hz}, 1 \mathrm{H}), 7.90(\mathrm{~d}, J=8.3 \mathrm{~Hz}, 1 \mathrm{H}) .{ }^{13} \mathrm{C} \mathrm{NMR}$ $\left(125 \mathrm{MHz}, \mathrm{CDCl}_{3}\right): \delta 14.6,14.8,20.5,22.0,22.2,32.5,36.9,39.1,124.6,125.5,126.3$, 126.4, 127.9, 128.0, 128.7, 129.1, 132.3, 132.3, 132.9, 133.8, 136.9, 138.9, 140.5. Anal. Calcd for $\mathrm{C}_{26} \mathrm{H}_{30}$ : C, 91.17; H, 8.83. Found: C, 91.17; H, 9.03.

The Reaction of $1 \mathrm{~b}$ with $2 \mathrm{a}$ in the Presence of a Stoichiometric Amount of Fe(acac) (Scheme 2). To a solution of $\mathrm{Fe}(\mathrm{acac})_{3}(53.0 \mathrm{mg}, 0.150 \mathrm{mmol})$ and $\mathrm{PBu}_{3}(243 \mathrm{mg}, 1.20 \mathrm{mmol})$ in THF $(5 \mathrm{~mL})$ was added a Et ${ }_{2} \mathrm{O}$-benzene $(1: 1)$ solution $(0.33 \mathrm{M})$ of 2-methylnaphth-1-ylmagnesium bromide (1b: $1.82 \mathrm{~mL}, 0.601 \mathrm{mmol})$ and the mixture was stirred for $10 \mathrm{~min}$ at room temperature. To this solution was added 4-octyne (2a: $16.5 \mathrm{mg}$, $0.150 \mathrm{mmol})$, and the mixture was stirred at $60^{\circ} \mathrm{C}$. After $20 \mathrm{~min}$, water $(10 \mathrm{~mL})$ was added and the resulting mixture was extracted with diethyl ether $(10 \mathrm{~mL} \times 3)$. The combined organic layer was washed with brine $(10 \mathrm{~mL})$, and dried over anhydrous magnesium sulfate. Evaporation of the solvent followed by purification with PTLC $\left(\mathrm{SiO}_{2}\right)$ gave (E)-4-(2-methylnaphth-1-yl)-4-octene (3b: $32.1 \mathrm{mg}, 85 \%)$ as a colorless oil.

The Reaction of $1 b$ with $2 a$ in the Presence of a Stoichiometric Amount of $\mathbf{C u B r}$ (Scheme 2). To a solution of $\mathrm{CuBr}(21.5 \mathrm{mg}, 0.150 \mathrm{mmol})$ and $\mathrm{PBu}_{3}(121 \mathrm{mg}, 0.598 \mathrm{mmol})$ in THF $(5 \mathrm{~mL})$ was added a $\mathrm{Et}_{2} \mathrm{O}$-benzene $(1: 1)$ solution $(0.33 \mathrm{M})$ of 2-methylnaphth-1-ylmagnesium bromide (1b: $1.82 \mathrm{~mL}, 0.601 \mathrm{mmol})$ and the mixture was stirred for $10 \mathrm{~min}$ at room temperature. To this solution was added 4-octyne (2a: $16.5 \mathrm{mg}$, $0.150 \mathrm{mmol}$ ), and the mixture was stirred at $60{ }^{\circ} \mathrm{C}$ for $24 \mathrm{~h}$. An aliquot of the resulting mixture was sampled and treated with diethyl ether- $\mathrm{H}_{2} \mathrm{O}$. GC analysis of the organic layer showed that it does not contain even a trace amount of (E)-4-(2-methylnaphth-1-yl)-4-octene (3b). 


\section{References}

(1) Hayashi, T.; Inoue, K.; Taniguchi, N.; Ogasawara, M. J. Am. Chem. Soc. 2001, 123, 9918-9919.

(2) Genin, E.; Michelet, V.; Genêt, J.-P. J. Organomet. Chem. 2004, 689, 3820-3830. 